\title{
ERRATUM: Adiponectin acts in the brain to decrease body weight
}

Yong Qi, Nobuhiko Takahashi, Stanley M Hileman, Hiralben R Patel, Anders H Berg, Utpal B Pajvani, Philipp E Scherer \& Rexford S Ahima Nat. Med. 10, 524-529 (2004)

Three sentences in this article appeared incorrectly. On p. 524, in the second paragraph after the abstract, the fifth sentence should read "ADP circulates in serum as low-molecular-weight dimers of trimers and high-molecular weight complexes 5 ." On p. 525, in the first paragraph in the right column, the second sentence should read “We also injected ADP $(2 \mu \mathrm{g} / \mathrm{g})$ or PBS i.v. and measured serum and CSF ADP.” In the second paragraph in the same column, several words were repeated in the second and third sentences; the second sentence should read "ADP (0.1 $\mu \mathrm{g} / \mathrm{g}$, i.c.v.) induced Fos-positive cells in the paraventricular nucleus (PVN; Fig. 2d)."

We regret the errors.

\section{ERRATUM: Depletion of host Langerhans cells before transplantation of donor alloreactive T cells prevents skin graft-versus-host disease}

Miriam Merad, Petra Hoffmann, Erik Ranheim, Sarah Slaymaker, Markus G Manz, Sergio A Lira, Israel Charo, Donald N Cook, Irving L Weissman, Samuel Strober \& Edgar G Engleman

Nat. Med. 10, 510-517 (2004)

TCD bone marrow was defined in the article as "T-cell-derived" bone marrow. The correct definition is "T-cell-depleted" bone marrow.

We regret the error.

\section{CORRIGENDUM: Direct evidence that the VEGF-specific antibody} \section{bevacizumab has antivascular effects in human rectal cancer}

Christopher G Willett, Yves Boucher, Emmanuelle di Tomaso, Dan G Duda, Lance L Munn, Ricky T Tong, Daniel C Chung, Dushyant V Sahani, Sanjeeva P Kalva, Sergey V Kozin, Mari Mino, Kenneth S Cohen, David T Scadden, Alan C Hartford, Alan J Fischman, Jeffrey W Clark, David P Ryan, Andrew X Zhu, Lawrence S Blaszkowsky, Helen X Chen, Paul C Shellito, Gregory Y Lauwers \& Rakesh K Jain Nat. Med. 10, 145-147 (2004)

The complete trial schema was omitted from the article. The protocol is outlined below.

Cycle 1: weeks 1 and 2

\begin{tabular}{|c|c|c|c|c|c|c|c|c|c|c|c|c|c|c|}
\hline & M & $\mathrm{Tu}$ & We & Th & $\mathrm{Fr}$ & $\mathrm{Sa}$ & Su & $M$ & $\mathrm{Tu}$ & We & Th & $\mathrm{Fr}$ & Sa & Su \\
\hline \multirow[t]{3}{*}{ BV } & $x$ & & & & & & & & & & & & & \\
\hline & \multicolumn{14}{|c|}{ Cycle 2: weeks 3 and 4} \\
\hline & $M$ & $\mathrm{Tu}$ & We & Th & $\mathrm{Fr}$ & Sa & Su & M & $\mathrm{Tu}$ & We & Th & $\mathrm{Fr}$ & Sa & Su \\
\hline BV & $x$ & & & & & & & & & & & & & \\
\hline $5-\mathrm{FU}$ & $x$ & $x$ & $x$ & $x$ & $x$ & $x$ & $x$ & $x$ & $x$ & $x$ & $x$ & $x$ & $x$ & $x$ \\
\hline \multirow[t]{3}{*}{ EBRT } & $x$ & $x$ & $x$ & $x$ & $x$ & & & $x$ & $x$ & $x$ & $x$ & $x$ & & \\
\hline & \multicolumn{14}{|c|}{ Cycle 3: weeks 5 and 6} \\
\hline & $M$ & Tu & We & Th & $\mathrm{Fr}$ & Sa & Su & M & $\mathrm{Tu}$ & We & Th & $\mathrm{Fr}$ & $\mathrm{Sa}$ & Su \\
\hline BV & $x$ & & & & & & & & & & & & & \\
\hline $5-\mathrm{FU}$ & $x$ & $x$ & $x$ & $x$ & $x$ & $x$ & $x$ & $x$ & $x$ & $x$ & $x$ & $x$ & $x$ & $x$ \\
\hline \multirow[t]{3}{*}{ EBRT } & $x$ & $x$ & $x$ & $x$ & $x$ & & & $x$ & $x$ & $x$ & $x$ & $x$ & & \\
\hline & \multicolumn{14}{|c|}{ Cycle 4: weeks 7 and 8} \\
\hline & $M$ & $\mathrm{Tu}$ & We & Th & $\mathrm{Fr}$ & $\mathrm{Sa}$ & Su & M & $\mathrm{Tu}$ & We & Th & $\mathrm{Fr}$ & $\mathrm{Sa}$ & $\mathrm{Su}$ \\
\hline BV & $x$ & & & & & & & & & & & & & \\
\hline $5-\mathrm{FU}$ & $x$ & $x$ & $x$ & $x$ & $x$ & $x$ & $x$ & $x$ & $x$ & $x$ & & & & \\
\hline EBRT & $x$ & $x$ & $x$ & $x$ & $x$ & & & $x$ & $x$ & $x$ & & & & \\
\hline
\end{tabular}

One cycle consisted of 2 weeks. Treatment was for a maximum of four cycles. Bevacizumab (BV) was delivered as intravenous infusion ( $5 \mathrm{mg} / \mathrm{kg}$ ) on day 1 of each cycle for four cycles (days 1, 15, 29, 44). 5- Fluorouracil (5-FU) was administered by an infusion pump over $24 \mathrm{~h}$ each day (at a fixed daily dose of $225 \mathrm{mg} / \mathrm{m}^{2} / 24 \mathrm{~h}$ ) throughout each treatment week of cycles 2-4. External beam radiation therapy (EBRT) was delivered at $1.8 \mathrm{~Gy} / \mathrm{d}, 5 \mathrm{~d} /$ week (excluding holidays) for a total radiation dose of 50.4 Gy in 28 fractions over a period of 6 weeks. Surgery was carried out 7-9 weeks after completion of preoperative bevacizumab, 5-FU and EBRT. All six patients completed therapy without dose-limiting toxicity. 